
du F.L.E. (18e et 19 e siècles)

\title{
Écrivains français de l'âge classique et traduction dans les manuels espagnols de la seconde moitié du $19^{\mathrm{e}}$ siècle
}

Ester Juan Oliva

\section{OpenEdition}

\section{Journals}

Édition électronique

URL : https://journals.openedition.org/dhfles/3026

DOI : $10.4000 /$ dhfles.3026

ISSN : 2221-4038

Éditeur

Société Internationale pour l'Histoire du Français Langue Étrangère ou Seconde

Édition imprimée

Date de publication : 1 décembre 1999

Pagination : p.223-231

ISSN : 0992-7654

Référence électronique

Ester Juan Oliva, «Écrivains français de l'âge classique et traduction dans les manuels espagnols de la seconde moitié du 19 e siècle », Documents pour I'histoire du français langue étrangère ou seconde [En ligne], 24 | 1999, mis en ligne le 04 février 2015, consulté le 27 mai 2021. URL : http:// journals.openedition.org/dhfles/3026 ; DOI : https://doi.org/10.4000/dhfles.3026

Ce document a été généré automatiquement le 27 mai 2021.

(c) SIHFLES 


\title{
Écrivains français de l'âge classique et traduction dans les manuels espagnols de la seconde moitié du $19^{\mathrm{e}}$ siècle
}

\author{
Ester Juan Oliva
}

1 On connaît l'importance fondamentale que revêt la traduction dans l'enseignement des langues étrangères au $19^{e}$ siècle en Espagne. On la retrouve, sous l'une ou l'autre forme, dans la majorité des méthodes, grammaires et manuels divers et le rôle que lui accordent les différentes réformes éducatives à travers le $19^{\mathrm{e}}$ siècle, confirme la place qu'elle occupe dans la réflexion des différents acteurs du monde éducatif. Cependant, nous ne traiterons pas ici des multiples fonctions pédagogiques potentielles de la traduction comme instrument partiel d'une méthodologie de l'enseignement du français langue étrangère, mais des quelques manuels qui, pendant ce demi-siècle, sont consacrés à la traduction en tant qu'objectif spécifique et de la place qui y est assignée aux écrivains français de l'âge classique.

2 Outre les collections de phrases et les quelques extraits que l'on trouve dans les manuels plus généraux, il faut également signaler que l'on apprend aussi à traduire sur base de recueils littéraires, tels que le célèbre Leçons françaises de littérature et de morale de Francisco Tramarria (Madrid, 1939). Son influence s'étend sur tout le $19^{\mathrm{e}}$ siècle et donc, à l'époque qui nous intéresse, comme nous le verrons plus loin. Bien entendu, l'objectif de cet ouvrage est plus large que la perspective particulière que nous adoptons ici et de fait, il ne donne aucune instruction particulière sur la traduction, de sorte que nous nous limiterons à signaler les commentaires que l'on trouve à son sujet chez les auteurs postérieurs.

3 Le nombre de manuels portant de manière plus précise sur la traduction est assez limité par rapport au nombre de manuels de français que l'on trouve pour cette période et dont la croissance considerable dans la deuxième partie du siècle inspire ce commentaire à Femando Araujo ${ }^{1}$ : 
Jamás hemos podido comprender la multiplicación verdaderamente pasmosa de los libros de texto, y singularmente de los destinados a la enseñanza del francés, en España, cuando esa multiplicación no aumenta ni en la más insignificante medida el caudal científico de nuestros conocimientos, ni añade en genera] un átomo al activo de nuestro balance intelectual. Tómese por ejemplo la Gramática de Chantreau por una parte y la de Tramarría por otra [...], y cotejándolas con el diluvio de Gramáticas y Métodos posteriormente publicados, dígase si estos últimos en general representarán el más mínimo progreso sobre aquellos y si no vienen a ser, con rarísimas excepciones, su más o menos fiel trasunto. / [Jamais nous n'avons pu comprendre la multiplication véritablement saisissante des manuels, et particulièrement de ceux destinés à l'enseignement du français, en Espagne, alors que cette multiplication n'enrichit pas un tant soit peu nos connaissances scientifiques, ni n'ajoute en général un atome à l'actif de notre bilan intellectuel. Prenons par exemple la grammaire de Chantreau d'un côté, et celle de Tramarría de l'autre [...], et si on les compare avec le déluge de grammaires et de méthodes publiés par la suite, qu'on constate si ces derniers peuvent pour la plupart représenter le moindre progrès par rapport aux premiers et s'ils n'en sont pas tout simplement, à de rares exceptions près, la plus ou moins fidèle reprise.] (1891: VII).

4 Les témoignages des situations éducatives diverses nous permettront d'approcher une pratique souvent entreprise dans des conditions difficiles par des professeurs parfois mal préparés qui tâchent de s'adapter et souvent, dans les introductions et avertissements des ouvrages qu'ils publient, de justifier de manière plus ou moins cohérente leurs choix.

5 Au début de cette seconde moitié du $19^{\mathrm{e}}$ siècle, l'enseignement des langues étrangères s'impose comme une nécessité dans l'enseignement secondaire. Cependant, le français, bien qu'il jouisse habituellement d'un statut privilégié par rapport à d'autres langues, continue à faire partie des matières considérées comme accessoires, au même rang que le dessin, par exemple. Son enseignement est confié à des professeurs sans formation spécifique et souvent marginaux au sein de l'institution éducative. La loi Moyano de 1857, apporte un changement dans la mesure où elle introduit dans l'enseignement secondaire l'étude (pendant deux ans) d'une langue vivante dont le choix sera déterminé par les possibilités de l'établissement. Cependant, la responsabilité de cette matière doit revenir à un agrégé de français ou de tout autre langue vivante, sans qu'il soit exigé dans le second cas de formation particulière, de sorte que la qualité de cet enseignement profite peu du changement ${ }^{2}$.

6 Dans ce contexte apparaît le premier manuel auquel nous nous intéresserons, Traducción gradual del francés, literal interlineal, gramatical y libre, de Vicente Alcober y Largo (Madrid, Imp. Manuel Minuesa, 1857), qui se présente comme «professeur de langues à Madrid» ${ }^{3}$. Mais il présente surtout son manuel comme une alternative à Tramarria, et il ne sera pas le seul à justifier son entreprise par la nécessite d'adapter le matériel utilisé à l'usage qu'on veut en faire. Il défend ainsi la publication de manuels spécifiques pour l'enseignement de la traduction:

En el día, dos son las obras de que generalmente se echa mano para la traducción del francés. El Telémaco y los trozos de Tramarria. Ambas son de un mérito extraordinario pero no muy apropósito para el objeto de la traducción; y bien podría asegurar, sin temor a equivocarme, que sus autores no los compusieron con la mira de instruir a los extranjeros en el idioma francés. / [A ce jour, deux sont les ouvrages auxquels on a généralement recours pour la traduction du français. Le Télémaque et les extraits de Tramarria. Tous deux ont une valeur extraordinaire mais sont peu appropriés pour à la traduction; et je pourrais affirmer, sans risquer 
de me tromper, que leurs auteurs ne les ont pas composés avec l'intention

d'enseigner aux étrangers la langue française.] (1857: Prólogo).

7 Alcober y Largo critique cette pratique en raison de l'inadéquation du niveau en ce qui concerne le premier ouvrage cité, à laquelle s'ajoute l'absence d'explications pour le second. Il suggère donc une typologie de textes adaptée aux différents niveaux de difficulté de traduction, en fonction de l'argument selon lequel les narrations, les descriptions, les définitions et les fables sont plus difficiles à traduire que les extraits portant sur la morale religieuse, la philosophie pratique, les discours, etc. Le critère qu'il adopte est en fait la facilité de la compréhension, à laquelle il superpose une progression dans la qualité de la traduction, pour laquelle il propose trois niveaux. Pour le premier, la traduction littérale interlinéaire, il propose un fragment du Petit catéchisme historique, dont il donne effectivement une traduction littérale, qu'il «arrange» au fil des notes où il suggère des solutions plus convenables, mais sans explications. Pour le second niveau, la traduction grammaticale à vue, il ajoute des extraits de l'Histoire ancienne à l'usage des maisons d'éducation. Finalement, l'élève doit atteindre la traduction libre, niveau le plus élevé, qui s'applique à des extraits en vers, presque toujours d'auteur français de l'âge classique, parmi lesquels Corneille (Le Cid, Héraclius, Cinna) et Racine (Iphigénie à Aulide, Bajazet, Mithridate) tiennent la vedette. Pourtant, la seule aide explicitement apportée à l'élève reste une série de notes proposant des solutions, souvent assez littérales, pour des mots ou tournures isolés. Il semble donc que l'acquisition $\mathrm{du}$ bon goût et du style élevé doive se faire indépendamment de cet exercice (au départ de modèles littéraires espagnols)... ou par osmose.

Quelques années plus tard, nous trouvons une édition de $1864 \mathrm{du}$ Cours de versions françaises ou choix de lectures graduées en prose et en vers, extraites des classiques français, avec de nombreuses notes, des appréciations et des notices bibliographiques d'Anselme Ouradou, professeur au Lycée de San Isidro, à Madrid. Ses préoccupations sont similaires à celles d'Alcober y Largo en ce qui concerne la programmation des exercices, encore qu'il rassemble à nouveau, comme l'indique le titre, les objectifs de lecture et de traduction. Outre l'établissement d'une typologie de textes comparable à la précédente, Ouradou s'inquiète d'assurer chez ses élèves la «parfaite morale» et la «noblesse des sentiments». On trouve donc dans son ouvrage des extraits de Fénelon, de Rousseau, ce dernier comme exemple des égarements moraux auxquels peut succomber un auteur de qualité. Sa collection d'extraits inclut également Fleury, Montesquieu, La Fontaine, Voltaire, Boileau... ainsi que des auteurs plus récents tels que Lamartine, dont il sélectionne les extraits concernant les thèmes annoncés.

9 Pour revenir à des considérations d'ordre linguistique, nous avons pu consulter également une seconde édition, de 1866, de l'Antigalicismo o sea libro de lectura francesa escogida, graduada y anotada, con el fin de evitar galicismos en la versión española, de Clemente Cornelias (Madrid), agrégé de français et d'anglais. Au-delà des considérations morales, il se pose la question du type de français qu'il faut enseigner. On passe ici à un tout autre objectif pour l'enseignement d'une langue étrangère:

...escribirlo y hablarlo como vulgarmente se habla y se escribe [...] ¿cómo se ha de lograr este objeto con trozos entresacados de obras escritas en estilo elevado y enfático [...]? Además, ¿qué se ha de sacar de trozos más que ideas imperfectas? / [la parier et l'écrire comme on la parle et l'écrit couramment [...] comment peut-on arriver à cela avec des extraits tirés d'œuvres de style élevé et emphatique [...]. En outre, que peut-on tirer d'extraits, si ce n'est des idées imparfaites?]. 
Il s'oriente donc vers des œuvres transcrites intégralement, de style simple, familier, etc., ajoutant malgré tout à ce critère la caution d'une vocation morale et sociale qui justifie les corrections de style et même de contenu à apporter aux textes originaux. Mais il opte résolument pour des auteurs contemporains tels Eugène Scribe, dont il donne des pièces complètes, écartant donc totalement les classiques au profit d'un enseignement plus efficace de la langue en tant que telle. A noter que Cornelias se propose également, comme l'indique son titre, de lutter contre le gallicisme:

¿Quién duda que la traducción de obras francesas al idioma patrio sin más guía que la escasa inteligencia del alumno, aunque auxiliada oralmente de la ayuda del profesor, es la causa de la corrupción de nuestro hermoso idioma, de la introducción en él del galicismo, que ya, por desgracia, ha invadido nuestra literatura / [Qui peut douter que la traduction d'œuvres françaises à notre langue sans autre guide que la faible intelligence de l'élève, bien que secourue oralement par le professeur, est la cause de la corruption de notre belle langue, de l'introduction du gallicisme, qui a malheureusement déjà envahi notre littérature.]

Contre cette imitation trop zélée des bons auteurs il arme ses élèves de notes concernant les mots ou tournures à risque.

Malgré une orientation de plus en plus «fonctionnelle» de l'enseignement du français, l'objectif d'acquérir un style élevé ne disparait cependant pas. On trouve ainsi $\mathrm{El}$ traductor francés o colección de obras escogidas de la literatura francesa en prosa y verso ordenadas y anotadas de Fernando García Ayuso (Madrid, $2^{\mathrm{e}}$ édition, 1883), également auteur de grammaires allemandes et arabes, ainsi que de différentes traductions ${ }^{4}$. Il rejoint Cornelias en ce qui concerne la nécessité de proposer des œuvres entières ou du moins des fragments suffisamment étendus (il critique explicitement Tramarria) mais rejette les choix de celui-ci, qu'il considère trop populaires et qui négligent la richesse de la littérature française:

Dista mucho de llenar este vacío un libro que, como el del Sr. Cornelias, se limita a reproducir obritas de tres autores de mediano gusto, y algunas de las cuales son además de dudoso gusto literario [...] / [Ce vide peut difficilement être comblé par un livre qui, comme celui de $\mathrm{M}$. Cornelias, se limite à reproduire les œuvres mineures de trois auteurs de goût moyen, et dont certaines sont d'un goût littéraire douteux [...].] (1883: V).

Retour donc aux classiques avec une séquence d'œuvres et de fragments ainsi articulée:

...empezando por los cuentos más sencillos en prosa y las fábulas más fáciles de entender en verso y acabando por el estilo clásico más elevado / [en commençant par les contes les plus simples en prose et les fables les plus faciles à comprendre en vers pour terminer par le style classique le plus élevé] (VI).

Le critère qu'il utilise est semblable à celui d'Alcober y Largo, bien qu'il diffère dans l'évaluation des différents types de textes. La sélection est accompagnée, cette trois, de notices bibliographiques. Elle inclut le Catéchisme historique de Fleury, des contes de Perrault, des textes de type pratique (par exemple, Les horloges) mais aussi Eugène Scribe qui récupère ici le statut d'auteur spirituel et d'excellent style. On trouve également l'Histoire universelle de Bossuet, Fénelon, La Bruyère, Mme de Sévigné, La Fontaine, Florian, Racine, Boileau ainsi que des auteurs plus récents tels que Chateaubriand et Lamartine. Malgré les critères de difficulté affichés, on constate qu'aucun critère chronologique n'intervient dans l'ordre de présentation des œuvres, qui aboutit à nouveau au style classique comme objectif ultime. 
15 En dépit de ce retour de flamme, le paysage éducatif a changé et bien que les multiples réformes ne soient pas toujours appliquées et que les ambitions qu'elles reflètent tardent à se concrétiser, les deux dernières décennies montrent un changement important dans les manuels consacrés à l'enseignement de la traduction du français. Les décrets d'application de la Loi Moyano avaient permis une certaine association du français avec l'élégance et la culture alors que d'autres langues, comme l'anglais ou l'allemand étaient associées à l'utilitaire. Une nouvelle réforme de 1866 réduit la présence de la langue étrangère à un examen de traduction donnant accès au statut de bachiller, ce qui laisse l'enseignement en tant que tel aux mains du secteur privé. En outre, un décret postérieur suspend la nomination d'agrégés de français et cela pendant près de vingt ans. L'enseignement des langues vivantes revient dans les programmes en 1880, avec la réforme de Lasala y Collado, qui abandonne la catégorisation des matières en «études générales» et «d'application» et s'oriente plus résolument vers l'identification de l'enseignement des langues étrangères avec la modernité (Calle, 1990: 184-186).

Dans ce sens, et quoique étranger en principe à l'objet de ce travail, il est intéressant de mentionner la Nueva gramática y trozos de traducción militares de Juan Ostenero y Velasco (Toledo, Faude e Hijo, 1882). Il s'agit d'un militaire qui exerce également comme professeur à l'Académie d'infanterie, et qui rejoint ce que l'on pourrait appeler aujourd'hui un profil professionnel. Ostenero se propose donc d'enseigner un français instrumental au service du progrès de l'armée, comme il l'explique dans son Prologue:

Por desgracia, muchos son los que hoy se lamentan de un descuido cuyas consecuencias no pudieron prever, y lo mismo ocurrirá mañana mientras no se preste la debida importancia a la asignatura de lenguas modernas. Antes se consideraron éstas como un mero adorno de educación; ahora es innegable que su estudio contribuye mucho al desarrollo intelectual y práctico de todas las profesiones [...] / [Malheureusement, nombreux sont ceux qui regrettent aujourd'hui une négligence dont ils n'avaient pas prévu les conséquences, et il en sera de même demain si l'on n'accorde pas à la matière de langues étrangères l'importance qui lui est due. On les considérait avant comme un simple rehaussement de l'éducation; il est aujourd'hui indéniable que leur étude contribue considérablement au développement intellectuel et pratique de toutes les professions [...].]

Cette volonté de centrer l'enseignement sur une spécialité pousse évidemment l'auteur à substituer aux classiques jusque là presque incontournables des textes de Bonaparte, Metternich, etc. (après des exercices préliminaires constitués de phrases et de tournures propres au domaine militaire).

18 Cette réflexion sur l'utilité des langues étrangères apparaît également dans la Gramática razonada historico-crítica de l'agrégé Fernando Araujo (Toledo/Madrid, Fernando Fe, 1891). Cette grammaire a pour complément des Ejercicios prácticos ainsi qu'une Colección de temas franceses para su traducción al castellano. Araujo se donne comme objectif de fournir à ses élèves une connaissance pratique qui leur permette de voyager et de faire $\mathrm{du}$ commerce sans recourir à un interprète, mais il rappelle aussi l'obligation figurant dans le Règlement de travailler la traduction. En référence aux chrestomathies et autres collections traditionnelles, il fait la remarque suivante:

... pero estas colecciones de trozos clásicos ofrecen también la dificultad de que, si bien es cierto que facilitan el conocimiento de las grandes obras literarias francesas, se hallan, por su lenguaje y por su estilo, harto distantes de la lengua contemporánea, siendo frecuentísimo que el alumno que ha logrado traducir a 
Boileau y a Montesquieu, a Racine y a Corneille, a Bossuet y a Massillon, no acierte a leer una noticia del Petit Journal ni a descifrar una carta, ni a sostener una pequeña conversación en francés [...] / [... mais ces collections d'extraits classiques posent également ce problème que, s'il est vrai qu'elles facilitent la connaissance des grandes œuvres littéraires françaises, ils sont très éloignés, par leur langage et par leur style, de la langue contemporaine, de sorte qu'il est très fréquent que l'élève qui a su traduire Boileau et Montesquieu, Racine et Corneille, Bossuet et Massillon, soit incapable de lire un article du Petit Journal ni de déchiffrer une lettre, ni maintenir une simple conversation en français [...].] (Colección de temas franceses para su traducción al castellano: Prefacio).

De là que sa collection de thèmes ait pour sous-titre Trozos selectos, anécdotas, diálogos, consejos, charadas, cartas, acertijos, chistes, problemas, etc. Et il n'hésite pas à souligner que les extraits classiques qui s'y trouvent ne sont qu'un complément. De fait, les classiques sont principalement présents dans cet ouvrage à travers des «petites phrases», une place de choix étant réservée aux Maximes de La Rochefoucauld.

Carlos Soler y Arqués, professeur au Lycée Cardenal Cisneros propose une approche personnelle dans ses Lecciones de lengua francesa (Madrid, éd. consultée de 1895), dont la seconde partie est consacrée à la Versión directa e inversa. Son manuel commence par un avertissement dans lequel il précise l'utilité des thèmes français et la fonction que remplissent les extraits d'auteurs classiques:

...han de servir de ejercicio para la lectura, la traducción directa y el análisis lexicográfico que constituye el fondo de nuestras Lecciones. Añadimos trozos escogidos y clásicos franceses que amplíen sobradamente los ejercicios, apuntando además advertencias útiles para facilitar una traducción perfecta. / [ils doivent servir à l'exercice de la lecture et à l'analyse lexicographique qui constitue le fond de nos Leçons. Nous ajoutons des morceaux choisis et des classiques français destinés à approfondir suffisamment les exercices et introduisons en outre des recommandations utiles qui faciliteront une traduction parfaite].

21 En ce qui concerne les modalités de traduction, cet auteur distingue comme certains de ses prédécesseurs, parmi lesquels Alcober y Largo, la traduction littérale, la traduction grammaticale et la traduction libre. Les arguments justifiant ces catégories sont exposés dans des «Règles générales à propos de la traduction». Ainsi, le premier type de traduction doit permettre l'analyse contrastive mais aussi la transmission intacte de textes sacrés tels que la Bible, le Coran ou les Védas. La seconde modalité, présentée comme étant moins servile sans cependant céder en rigueur convient aux œuvres didactiques, aux textes judiciaires, aux contrats, etc. On remarque qu'aux typologies de textes traditionnelles viennent s'ajouter dans cet ouvrage des textes non littéraires associés au commerce ou à l'exercice de diverses professions. Enfin, la traduction libre

...hace completa abstracción de las ideas y de los pensamientos [...]. Ordinariamente debe ser libre la traducción de las composiciones jocosas, altamente poéticas o novelescas y, en general, todas las de estilo muy familiar o muy elevado (1895: 6) / [fait totalement abstraction des idées et des pensées [...]. Il faut en principe traduire librement les compositions comiques, hautement poétiques ou romanesques et, de manière générale, toutes celles dont le style est très familier ou très élevé].

22 L'auteur applique les différentes modalités de traduction qu'il expose à des fragments traduits intégralement en guise de modèles. Pour la traduction libre, qui concerne les fragments d'auteurs classiques mais aussi contemporains allant de Buffon, Molière, Boileau, etc. à Chateaubriand ou Musset, il propose comme modèle la traduction d'un fragment du Gil Blas de Lesage. Une fois de plus, la traduction libre est associée au style 
le plus élevé, mais cette combinaison n'est plus présentée comme le seul objectif souhaitable.

L'intérêt croissant pour l'aspect utilitaire des langues étrangères et plus particulièrement $d u$ français s'étend donc aux exercices de traduction et c'est également le cas dans les Ejercicios de Traducción francesa (Valladolid, Santaren, 1897) d'Angel María Alvarez Taladriz. En effet, son manuel ne se fixe pas uniquement comme objectif celui que son titre suggère, à savoir apprendre à traduire, mais aussi celui de réviser d'autres matières du cursus, telles que l'histoire universelle et la géométrie. Les textes proposés pour la traduction, qui vont des fragments d'histoire (sans références) à la définition du polygone, par exemple, ne sont accompagnés ni de notes ni d'orientations particulières. La réflexion sur la traduction n'apparaît donc explicitement que dans l'un des textes proposés comme exercice et qui est d'ailleurs le seul classique repris ici: un fragment de De la manière d'enseigner et d'étudier les belles lettres de Charles Rollin, qui prêche le juste milieu entre la traduction libre et la traduction littérale. Comme nous l'avons signalé, ce manuel, qui est le dernier dont nous parlerons, remplace Fénelon par la géométrie pure et dure, la volonté d'éduquer au sens large cède la place à l'occasion de rentabiliser un apprentissage linguistique.

Ce petit itinéraire n'a d'autre ambition que de mettre en lumière l'une des facettes de l'évolution de la représentation des classiques français dans l'enseignement du français en Espagne à la fin du $19^{e}$ siècle. Bien que cette vision soit trop partielle pour en tirer des conclusions très tranchées, elle illustre assez clairement la mise à l'écart progressive des classiques au profit d'un enseignement des langues étrangères envisagé comme tel et de plus en plus explicitement présenté comme la voie d'accès à la modernité. Au départ objectifs fondamentaux de l'apprentissage d'une langue elle même considérée comme secondaire par rapport à d'autres matières du cursus, ils deviennent les objectifs très secondaires de l'apprentissage d'un français de plus en plus fonctionnel, qui les exclut de ce que l'on appellerait aujourd'hui les «besoins langagiers».

\section{NOTES}

1. Araujo, $\mathrm{F}\left(1891,2^{\mathrm{e}}\right.$ édition): Gramática razonada histórico-crítica de la lengua francesa, ToledoMadrid.

2. Voir Calle Carabias, Q. (1990): La enseñanza de idiomas en España, tesis doctoral. Málaga, pp. 173-175.

3. Il est en effet l'auteur de manuels similaires pour l'anglais, l'italien et l'allemand.

4. Le manuel inclut d'ailleurs les tarifs des traductions réalisées par les professeurs de l'Académie où il exerce et qui fonctionne donc également comme «agence» de traduction. 


\section{AUTEUR}

ESTER JUAN OLIVA

UNED, Madrid 